\title{
Limb Segment Recruitment as a Function of Movement Direction, Amplitude, and Speed
}

\author{
Joost J. Schillings \\ Ruud G. J. Meulenbroek \\ Arnold J. W. M. Thomassen \\ Nijmegen Institute for Cognition and Information \\ Nijmegen, The Netherlands
}

\begin{abstract}
Coordination of limb segments in graphic motor behavior has been studied primarily in cyclic tasks. In the present study, limb segment recruitment patterns were investigated in a discrete line-drawing task. Subjects $(N=11)$ performed pointing movements varying in direction, amplitude, and speed. The contributions of index finger, hand, and arm to the movement were analyzed by evaluating the angular displacements in 7 joint dimensions. The results showed that amplitude and direction affected limb segment involvement in the same way they have been reported to affect it in cyclic movements. Upward left- (up-left) directed movements were primarily achieved by fingers and arm, whereas upward right- (up-right) directed movements were accomplished with the hand and the arm. Large amplitudes elicited not only an increase of proximal but also a decrease of distal limb segment involvement, especially in the up-left direction. In the present discrete pointing task, effects of speed on limb segment involvement were different from speed effects that were observed earlier in cyclic tasks: Larger limb segments became more involved in fast than in slow discrete movements. With respect to the timing of limb segment recruitment, all joints tended to move simultaneously, but small deviations from synchronous joint movement onset and offset were present. The results are discussed in the context of recent theories of limb segment coordination.
\end{abstract}

Key words: coordination, drawing, human arm movements, timing

n drawing and writing, as well as in most other kinds of motor behavior, we have more degrees of freedom (Bernstein, 1967) available at the level of limb segment coordination than are strictly necessary for performing the tasks successfully. For example, to move the tip of a pen to a certain position in space, a person holding that pen would need only three degrees of freedom. The human arm, however, even when the fingers and thumb are constrained to hold a pen, has many more degrees of freedom at the joint level.
The redundant degrees of freedom allow for a flexible way of movement selection in response to current environmental demands (Rosenbaum, Loukopoulos, Meulenbroek, Vaughan, \& Engelbrecht, 1995). But these demands are usually not strong enough to restrict the set of possible movements to a single solution. Thus, the actor is confronted with a degrees-of-freedom problem and has to choose one solution, often from many possible ones. An important question for motor control theories is how this choice is made.

To explain how people select movements, investigators have proposed several approaches. First, the number of degrees of freedom that one must actually control may be effectively reduced by maintaining dependencies between limb segments (Turvey, 1990). In graphic motor behavior, coupling of elbow and shoulder has been observed in circular drawing movements; during these movements, subjects tended to realize constant phase relations between these joints (Soechting, Lacquaniti, \& Terzuolo, 1986; Van Emmerik \& Newell, 1990). This outcome is consistent with the hypothesis that there are dependencies between limb segments.

Another approach to studying the way in which redundant degrees of freedom are dealt with is to identify efficiency constraints that may reduce the number of behavioral options. Such constraints may induce the selection of those limb segments that are most suitable to perform a task

Correspondence address: J. J. Schillings, Nijmegen Institute for Cognition and Information, P. O.Box 9104, 6500 HE Nijmegen, The Netherlands. E-mail address: SCHILLINGS@NICI.KUN.NL 
or they may limit the range of appropriate joint angles with which the task can be performed successfully. Efficiency constraints related to optimal amplitudes and frequencies of motion and optimal suitability for movement in a certain direction have been shown to affect the involvement of limb segments in repetitive pointing and drawing tasks (Meulenbroek, Rosenbaum, Thomassen, \& Schomaker, 1993; Rosenbaum, Slotta, Vaughan, \& Plamondon, 1991). In these studies, the relative contributions of fingers, hand, and arm to back-and-forth movements depended on direction, amplitude, and frequency of the movements.

Although efficiency constraints and dependencies that are expressed in terms of frequencies and phase relations are meaningful in describing cyclic phenomena, it is not obvious that they are also applicable to discrete, noncyclic movements. Perhaps movement speed in discrete movements is the logical equivalent of movement frequency in cyclical movements. But the question remains whether frequency-based efficiency constraints can be translated directly to speed-based ones and still be relevant. During high-frequency oscillating movements, it may be efficient to favor limb segments whose preferred frequency is high (Rosenbaum et al., 1991). During a high-speed discrete movement, however, the involvement of these segments may pay off less. Instead, other constraints may become more important, so that other segments become involved. Similar differences may be expected when considering phase relations between joints. A priori, it seems to be less relevant to look for fixed phase relations between joint angles in discrete movements than to look for them in cyclic movements; this relevance is larger, of course, to the extent that even discrete movements may be assumed to be (damped) oscillations (Guiard, 1993). In either case, however, and especially with respect to timing, it is still unclear how limb segments are coordinated in discrete movements and whether or not their involvement is synchronized to the same extent as in cyclic movements.

Studies focusing on limb segment coordination in the execution of discrete movements have thus far been based primarily on tasks involving pointing in a horizontal or vertical plane, during which the arm was constrained to move within that plane (e.g. Cruse, Brüwer, \& Dean, 1993; Kaminski \& Gentile, 1986). The joints that have been examined in these studies were shoulder, elbow, and wrist. In the present study, we investigated the contribution of the limb segments of the forearm in more detail by analyzing three-dimensional angular displacements of forearm, hand, and fingers. We paid special attention to the timing of these distal limb segments. In studies of cyclic movements, timing of limb segment actions has been considered in terms of phase relations. Little work has been dedicated to the issue of timing in discrete movements, although one study (Kaminski \& Gentile, 1986) reported some effects of amplitude variation on the relative timing of shoulder and elbow movements.

In the present experiment, we addressed two of the above-mentioned aspects of limb segment coordination in discrete movements. First, the findings by Meulenbroek et al. (1993) concerning the effects of direction, distance, and speed of end-effector displacement on limb segment involvement in cyclic drawing movements were compared with those obtained in a discrete drawing task. Our primary aim was to find out whether the efficiency constraints that were proposed in that study apply only to cyclical movements or to discrete ones as well. The second aspect of our study concerned the timing of limb segment movements. The question here was: What are the principles that govern the timing of the recruitment of limb segments in a discrete pointing task in which we do not expect fixed couplings of particular joints?

In contrast to earlier studies of limb segment coordination, we examined a drawing task in which the end effector, that is, the pen tip, moved in a single horizontal plane but the arm could move freely in space. We analyzed joint angular velocities, from elbow to index finger, to investigate both involvement of fingers, hand, and arm and timing aspects of their recruitment.

\section{Efficiency Constraints on Limb Segment Selection}

Meulenbroek et al. (1993) suggested that selection of limb segment patterns during cyclic drawing depends, among other things, upon optimal frequencies and amplitudes of motion of the effector segments that can contribute to the execution of the task. In their study, subjects performed back-and-forth movements of gradually increasing or decreasing size. Four different movement directions and three different maximum amplitudes were used. Working pace, instructed to be comfortable, slow, and fast, resulted in movement frequencies that were medium, low, and high, respectively. It turned out that the contribution of the forearm and the upper arm increased as the largest amplitude of the movements increased, whereas the contributions of the fingers and the hand decreased. Small but discernible effects of movement frequency were also present: The contribution of the fingers was large in high movement-frequency conditions, the contribution of the hand was large in medium movement-frequency conditions, and the contribution of the forearm and the upper arm tended to be large in low movement-frequency conditions. These ordinal relationships are in agreement with principles of mechanics if each segment is viewed as a linear damped oscillator (French, 1971); the observed optimal amplitude-frequency combinations can then be attributed to the length and mass of the limb segments, which determine their moment of inertia (see Rosenbaum et al., 1991).

Because the moments of inertia of the limb segments are particularly important in the initial acceleration and final deceleration of limb segments, we hypothesized that length and mass of the segments may also play a role in efficiency constraints influencing limb segment coordination during discrete movements. For example, in isolated pointing movements that start and finish with zero velocity, an efficiency constraint may be the minimization of the energy 
required to get the system going and to bring it to a full stop (Rosenbaum et al., 1995). The existence of such a constraint should result in an ordinal relationship comparable with the one mentioned above: As required movement speed gets higher, one would expect the contribution of low-inertia segments to the movement to increase, whereas high-inertia segments should be less involved, thus minimizing required energy. In the present study, we investigated whether such relations exist in the recruitment pattern of arm, hand, and fingers in fast and slow isolated graphic movements.

The hypothesis of Meulenbroek et al. (1993) concerning a similar relation between the amplitude of the end-effector displacement and the involvement of limb segments should also be applicable in the case of discrete movements. In discrete movements, it is not immediately clear how optimal amplitudes are related to optimal frequencies (Kay, Kelso, Saltzman, \& Schöner, 1987; Rosenbaum et al., 1991), because movements have to be produced in isolation. However, an efficient strategy might be to favor the use of limb segments with low moments of inertia as much as possible. Only when end-effector displacements become too large to be achieved primarily by those segments should there be increased involvement of limb segments with larger moments of inertia. Such a strategy would predict for pointing movements the ordinal relationship that was also observed in cyclic movements: The contribution to pen-tip displacement by the distal (low-inertia) segments decreased, whereas the contribution by proximal (high-inertia) segments increased as the amplitude of pen-tip displacement increased. In the present study, we tested this hypothesis by using two amplitude conditions.

Another factor that has been shown to affect limb segment selection in drawing is the orientation of the axis along which the pen tip is moved. Meulenbroek and Thomassen (1991) showed that right-handed subjects producing graphic movements in the horizontal plane, at table height, primarily used their fingers to produce movements along the line between upper left and lower right, as long as the amplitude of the movement was not too large. Movements along the line between upper right and lower left were accomplished primarily with the hand. It was also found that when amplitudes became too large to be achieved by the fingers or the hand, subjects respectively translated and rotated the forearm to accomplish the task in the above directions. Because these phenomena result from the orientation of the principal axes of rotation of the limb segments, given a normal writing posture, and not from the cyclic character of the task, we expected them to be replicated in the present study.

\section{Timing of Limb Segment Movements}

Little is known about the timing of limb segment recruitment in discrete pointing tasks. Several control principles for the timing of limb segment movements have been suggested recently by Rosenbaum et al. (1995). One of these is that each limb segment involved in the movement has its own preferred movement time. This would imply that, in general, different limb segments have different movement times, originating from the mechanical characteristics of the segments and the required angular displacement for their joints. It is an open question how, in this case, joint movements could be coordinated (e.g., by a constraint to start at the same time or stop at the same time). One study (Kaminski \& Gentile, 1986) reported that in horizontal planar pointing movements, shoulder and elbow had, in fact, different movement times. As a coordination principle, it was suggested that these joints tend to stop moving simultaneously, although they do not necessarily start at the same time. Another suggestion by Rosenbaum et al. (1995) was that, during their combined movement, all segments have the same movement times. These movement times could be externally imposed or could constitute a compromise between the individual preferred movement times of the joints. The idea of complete synchrony of limb segment motions (which would result in congruent joint velocity profiles) provides a simple timing mechanism that has the additional advantage that it ensures a straight-line movement through joint space, thus satisfying the principle of least action for motion through joint space (Rosenbaum et al., 1995). Because few empirical data exist that strongly favor either of the above-mentioned timing hypotheses, we investigated the timing of the onset and offset of angular displacements of joints in the present task to gain more insight into the timing aspects of limb segment coordination.

To summarize the main goals of the present experiment, we wanted to investigate whether, in spite of the differences between discrete and cyclic movements, the efficiency constraints that have recently been established for the latter movement type can also be observed in the former. Furthermore, we tried to find out the ways in which the movement onset and offset times of different joints relate to each other.

\section{Subjects}

\section{Method}

Eleven right-handed subjects ( 4 men, 7 women; ages 17-27) took part in the experiment. They were paid for their participation. Subjects participated only if their habitual pen grip involved holding the front end of the pen with the tip of the thumb, index finger, and middle finger, whereas the barrel of the pen rested on the hand, close to the metacarpophalangeal joint.

\section{Apparatus and Data Collection}

Subjects sat at a normal table with five red light-emitting diodes (LEDs; 4-mm diameter) built into its surface. These LEDs were controlled by a personal computer (i468/50) that also controlled the data collection. The LEDs were covered with a sheet of paper (format A4) with four preprinted target areas, as represented by the gray circles in Figure 1. The crosses indicate the position of the LEDs beneath the paper. The LED indicating the starting position (S) was located 32 $\mathrm{cm}$ from the near edge of the table, close to the body midline 


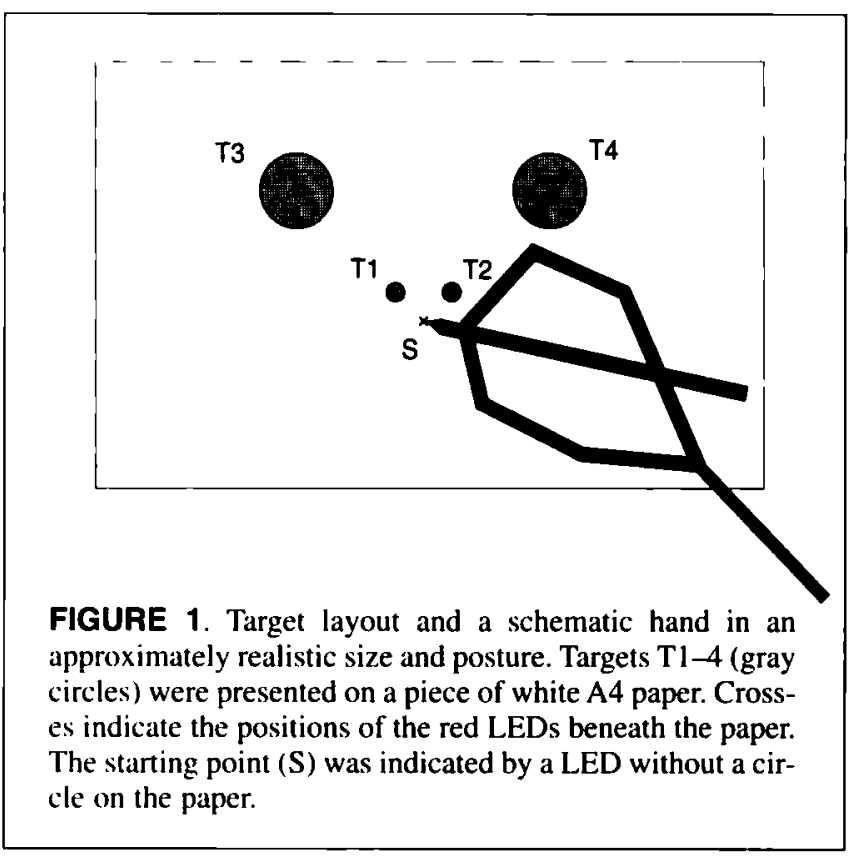

of the subject. The distances between starting point and the centers of the four target areas were $1.7 \mathrm{~cm}$ for the near targets (TI, T2) and $7.6 \mathrm{~cm}$ for the far targets (T3, T4). The widths of the target areas were 0.7 and $3.2 \mathrm{~cm}$, respectively. Target widths were chosen so that the index of difficulty for movements to the far targets was equal to that for movements to near targets (Fitts, 1954). Thus, for both amplitudes, the index of difficulty was 2.25 . On top of the sheet of paper was a transparent hard plastic board $(150.0 \times 75.0 \times$ $0.3 \mathrm{~cm}$ ) that covered the entire table and provided a smooth writing surface. Subjects used a felt-tipped pen that was dried out so that it did not leave traces on the surface. We attached a cotton cloth to the bottom side of the subjects' bare forearm to reduce its friction with the writing surface.

During the experiment, pen-tip displacements and finger, hand, and arm movements were recorded by means of an Optotrak 3020 system consisting of three infrared sensors. Seventeen infrared light-emitting diodes (IREDs) were used. Displacements of these IREDS were recorded with a sampling rate of $100 \mathrm{~Hz}$ and a spatial accuracy of $0.2 \mathrm{~mm}$.

Fourteen IREDs were attached to the subject's right arm, hand, and index finger, as depicted in Figure 2. We mounted IRED pairs $(4,5 ; 6,7 ; 8,9 ; 11,12$; and 13,14$)$ on small, hard plastic strips, both for ease of attachment and to eliminate relative IRED displacements within each marker pair as a result of skin displacements relative to the subjects' bone structure. The strip at which IREDs 13 and 14 were mounted was attached to the second phalanx of the index finger at a $90^{\circ}$ angle, such that the strip was pointing upward when the hand lay flat on the table. This ensured IRED visibility for the camera when the subject adopted a writing posture. Three IREDS were mounted at the top of the barrel of the pen (see Figure 2). They constituted an equilateral triangle with sides of $1.2 \mathrm{~cm}$, defining a plane parallel to the longitudinal axis of the pen. The position of each of these IREDS relative to the pen tip was calibrated after mounting it on the pen. The IRED layout described above constituted a number of virtual lines and planes, on the basis of which we estimated the angular displacement over time of four joints and the linear displacement over time of the pen tip.

In the laboratory, the table was positioned such that the camera was at a distance of approximately $2.50 \mathrm{~m}$ in front of the subject's arm. The focal point of the camera system was in the center of the subject's workspace, the line of sight making an angle of $45^{\circ}$ with the table surface. By using this set-up, we achieved an optimal IRED visibility for writing and drawing tasks.

\section{Procedure}

To test the hypotheses about the effects of direction, amplitude, and speed of movement on limb segment contribution, each subject performed eight variations of unidirectional graphic pointing movements. The direction of movement was

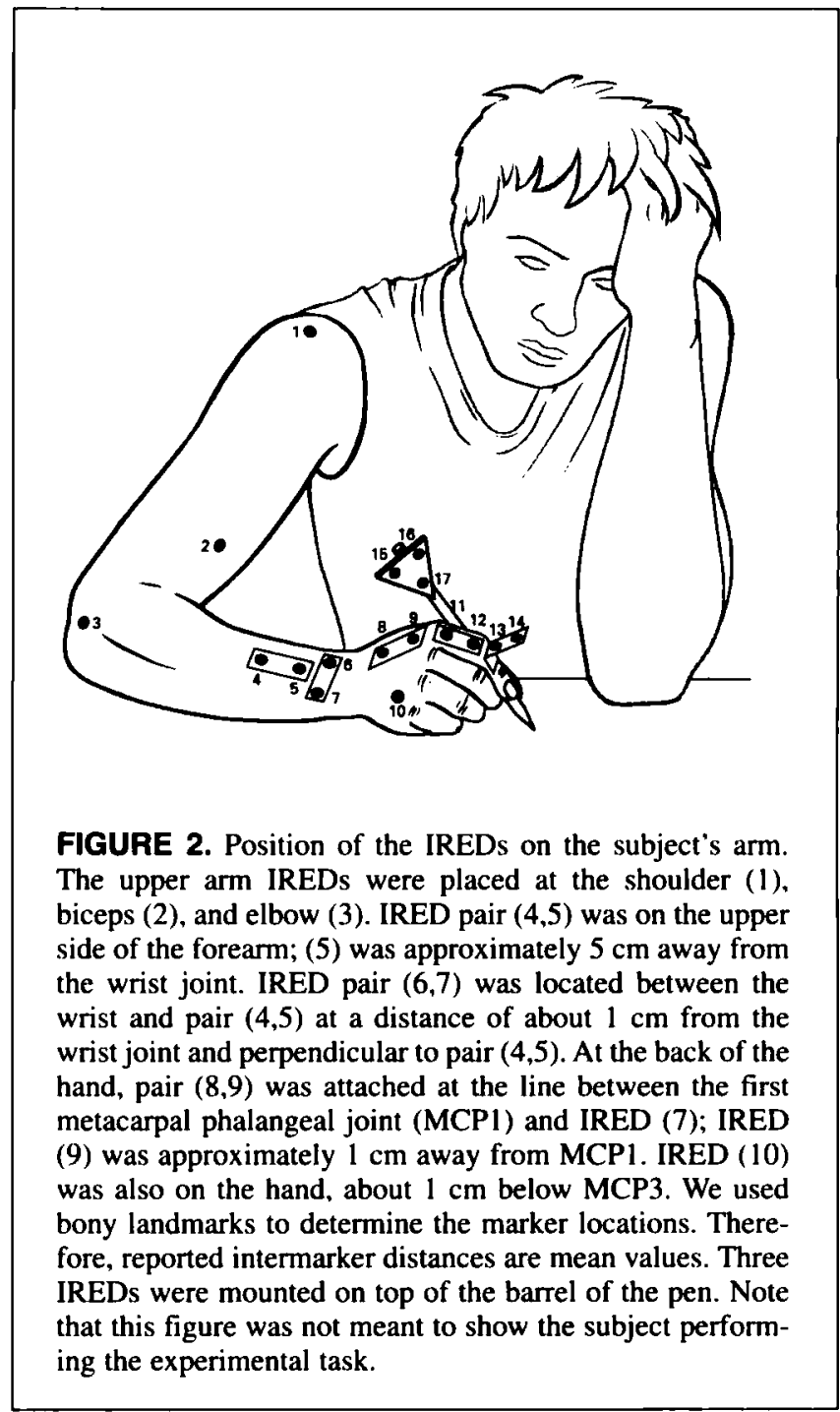


either to the upper left side (T1 and T3; see Figure 1) or the upper right side (T2 and T4) of the starting position. The amplitude of the movement was either small (approximately $1.7 \mathrm{~cm}, \mathrm{Tl}$ and $\mathrm{T} 2$ ) or large (approximately $7.6 \mathrm{~cm}$, targets T3 and T4). The instructed speed of movement was either slow (instructed movement time of $\mathbf{4 0 0} \mathrm{ms}$ ) or fast (instructed movement time of $200 \mathrm{~ms}$ ). Each subject performed two blocks of trials. In the first block, movement time was instructed to be $400 \mathrm{~ms}$; in the second block, instructed movement time was 200 ms. Within each block, 40 replications of four direction-distance combinations were performed in a random order, resulting in $\mathbf{3 2 0}$ trials per subject.

For each trial, the procedure was as follows. An acoustic low-frequency warning signal generated by the $\mathrm{PC}$ initiated the trial. Subjects were instructed to place the pen tip at the starting point and to adopt a comfortable writing posture upon presentation of the warning signal. After a foreperiod of $3 \mathrm{~s}$, one of the four target areas was indicated by lighting up the LED beneath it. At the same time, a high-frequency acoustic "go" signal was presented. Subjects were told to move the pen tip without lifting it and without interruptions, to the target area, immediately after perceiving the go signal. The duration of the go signal indicated the required movement time. Subjects had to finish their movement at the time the go signal was turned off. Before the start of the actual experiment, subjects performed 24 practice trials in which they were to tune their movement durations to the go signal durations in both speed conditions. In these practice trials, they received feedback on the difference between the required and the realized movement durations. At the end of the practice series, the subjects' movement speed satisfactorily agreed with the go signal duration. Taking into account an estimated average reaction time of $200 \mathrm{~ms}$, we used go signal durations of 600 and $400 \mathrm{~ms}$ to elicit movement times of approximately 400 and $200 \mathrm{~ms}$, in the two blocks. The pen had to remain at the end position in the target area until $3 \mathrm{~s}$ had passed since the go signal, that is, when the warning signal for the next trial was presented. Data collection started at the moment the go signal and the target light were presented and stopped $2 \mathrm{~s}$ later.

\section{Data Analysis}

From the three-dimensional IRED-displacement data, the following eight movement functions were derived:

1. Flexion and extension of the elbow

2. Pronation and supination of the forearm

3. Flexion and extension of the hand

4. Adduction and abduction of the hand

5. Flexion and extension of the first phalanx of the index finger

6. Adduction and abduction of the first phalanx of the index finger

7. Flexion and extension of the second phalanx of the index finger

8. Pen-tip displacement
Therefore, in addition to pen-tip displacements, we recorded excursions of four limb segments in one or two dimensions per segment, yielding angular displacement data in a total of seven joint dimensions (see Appendix). These angular signals, as well as the linear displacement signals of the pen tip, were filtered by using a second-order low-pass filter with a transition band of 8-14 Hz. After filtering, we differentiated all signals to obtain velocity profiles of the movements (see Figure 3). Flexion, adduction, and pronation resulted in positive angular velocities, whereas extension, abduction, and supination yielded negative angular velocities. The beginning and end of the pen-tip displacement were defined by local velocity minima in the tangential velocity profile. In this way, any small oscillations of the pen tip occurring when the movement came to a halt (because of elasticity of the effector system together with the relatively low friction between pen and writing surface) were ignored. Figure 3 depicts the pen-tip path and corresponding velocity profiles of the pen tip and the angular velocities in three of the seven joint dimensions (flexion or extension of the elbow, wrist, and second phalanx of the index finger) for one trial.

Movement time (MT) was defined as the duration of the pen-tip movement, that is, the time between the local minima defining its start and stop. As an index of motion in a certain joint dimension, we determined the sign-normalized peak angular velocities of the corresponding joint excursion. The higher the peak velocity, the larger the contribution of a limb segment to the pen-tip displacement was assumed to be.' This particular measure of contribution has the advantage of being fairly insensitive to noise, ${ }^{2}$ because at peak velocity, the signal-to-noise ratio is highest. To study the effects of the experimental variables on limb segment recruitment, we investigated the peak velocity variations in all joint dimensions. Sign-normalized peak angular velocities were averaged across the $\mathbf{4 0}$ replications per condition and used as cell entries in an analysis of variance (ANOVA) according to an 11 (subject) $\times 2$ (speed) $\times 2$ (direction) $\times 2$ (amplitude) factorial design.

For the analyses of the timing aspects of coordination, we determined, for each joint dimension, the moment at which the joint started to move, the moment at which it reached its peak velocity, and the moment at which it stopped moving. The moment at which peak joint velocities occurred could be determined reliably, but, because of noise, the moments at which the joints started and stopped moving required additional procedures. On the basis of a preliminary analysis of joint velocity variability in the absence of end-effector displacement, we selected an absolute joint velocity level of $1 \%$ to find the beginning and end of joint motion. In the absence of pen-tip displacement, the mean amplitude of the noise for all joint velocity profiles was smaller than $1 \%$. Note that this level does not guarantee that artifacts caused by soft tissue deformations were ruled out, because the arm was not moving during determination of this noise level. We started our search for level crossings by considering the points in time at which the pen point started and 


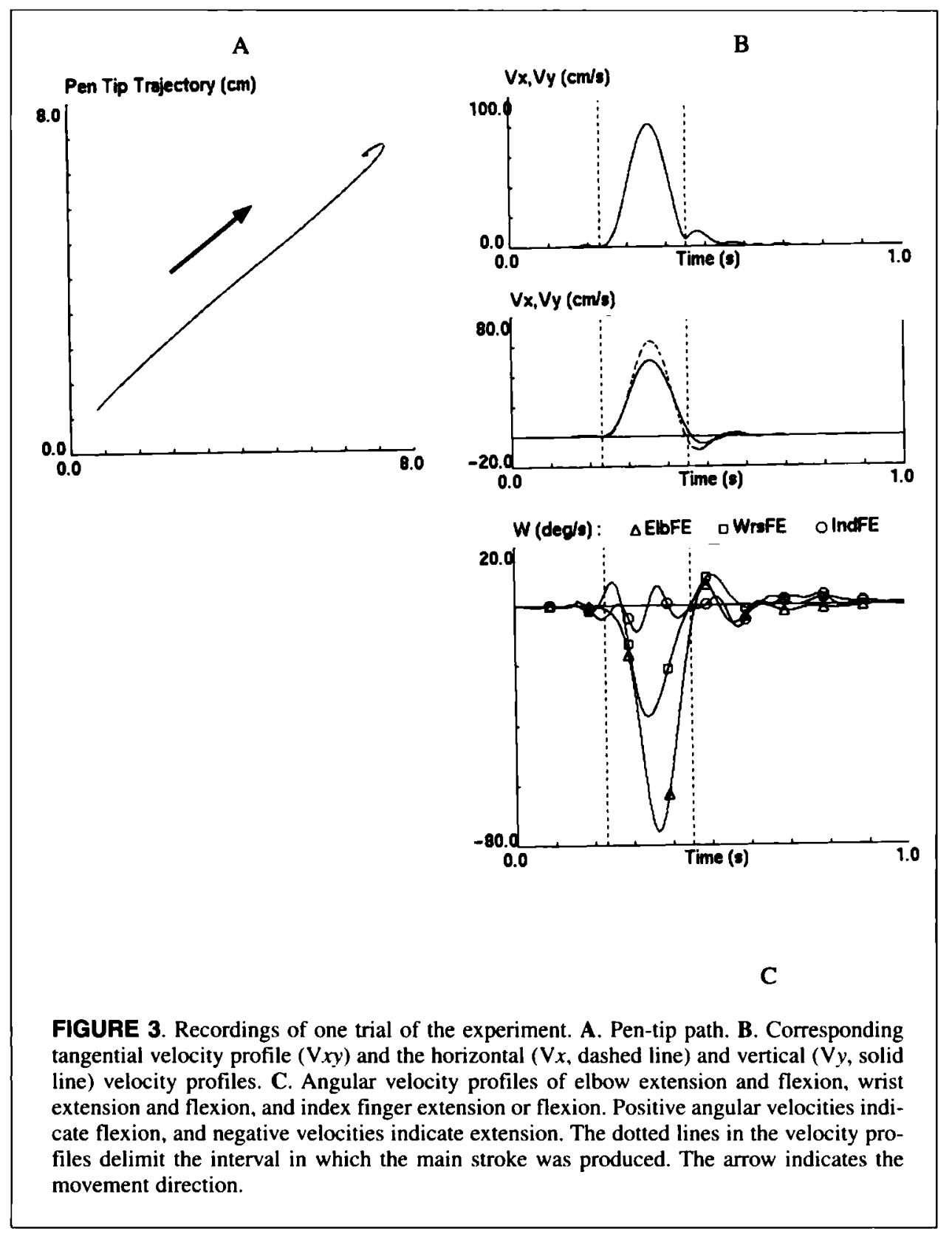

stopped moving, in an absolute sense. The $1 \%$ s level crossings that were nearest to these points, found either forward or backward in time and after interpolating between samples, were marked as the start and end points of the corresponding joint's movement. The latter points in time were adopted in our analysis.

Subsequently, for each joint dimension, we defined onset delay by subtracting the moment at which the pen tip started moving from the moment at which the joint started moving in that joint dimension. Peak and offset delays were defined analogously. Positive delays indicated that joint motion lagged behind pen-tip displacement, negative delays indicated that joint motion preceded pen-tip displacement. Onset, peak, and offset delays of the joints were averaged across the 40 replications and analyzed in an ANOVA with an 11 (subject) $\times 2$ (speed) $\times 2$ (direction) $\times 2$ (amplitude) $\times 7$ (joint dimension) design.

\section{Results}

\section{Subjects' Performance}

Subjects performed accurately with respect to the instructed movement amplitude and direction. The total pentip displacement, computed by integrating the tangential velocity profiles, was $1.95 \mathrm{~cm}(S D=0.39)$ for small movements and $7.80 \mathrm{~cm}(S D=0.75)$ for large movements. These distances were slightly larger than the distance between the starting point and the center of the corresponding target area ( 1.7 and $7.6 \mathrm{~cm}$, respectively), but this was caused by the 


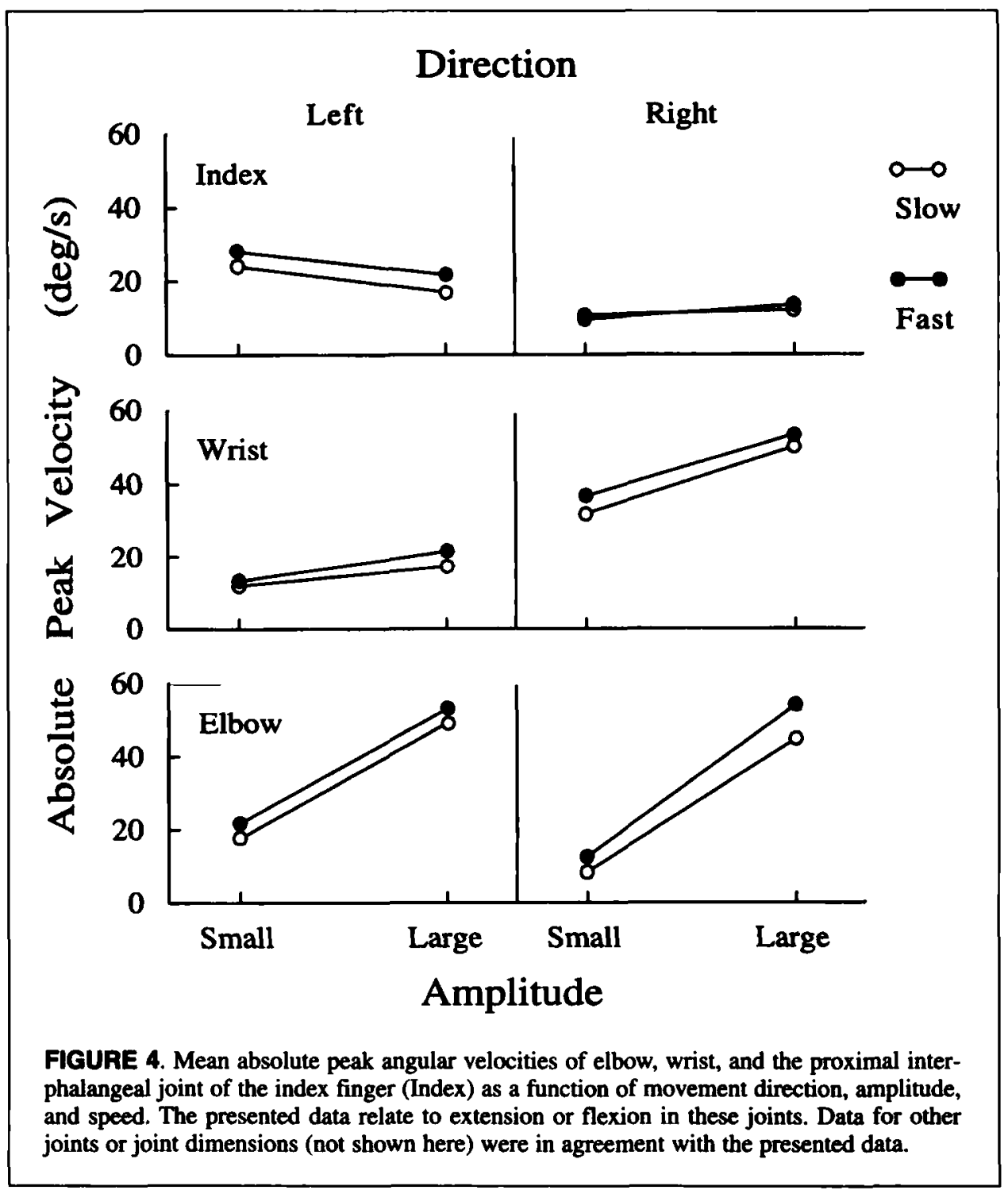

fact that pen-tip paths always deviated slightly from the straight line between the starting point and the end point of the movement. The net absolute pen-tip displacements in the $y$ direction were $1.32 \mathrm{~cm}(S D=0.27)$ and $5.32 \mathrm{~cm}(S D=$ 0.55 ) for small and large movements, respectively. For the $x$ direction, these values were $1.34(S D=0.34)$ and $5.59 \mathrm{~cm}$ $(S D=0.62)$. Given that both the horizontal and vertical distances to the corresponding target centers were 1.2 and 5.4 $\mathrm{cm}$ and that target widths were 0.7 and $3.2 \mathrm{~cm}$, this means that virtually all movements ended well within the target area. As can be seen in Figure 1, the target centers for leftward movements were located $135^{\circ}$ counterclockwise with respect to the positive $x$ direction, whereas target centers for rightward movements were located at $45^{\circ}$. On average, the line between the recorded start and end points of the movements was rotated $136.0^{\circ}$ ( $S D=5.75$ ) counterclockwise with respect to the positive $x$-axis in leftward movements, and $44.23^{\circ}(S D=3.05)$ in rightward movements. Thus, both amplitude and direction of the recorded movements were in good agreement with the instructions.

However, the movement times showed that subjects had trouble adhering to the instructed movement duration. We will come back to this when we consider the effects of movement speed.

\section{Effects of Movement Direction and Amplitude on Joint Excursions}

Figure 4 shows the mean sign-normalized peak angular velocities for the elbow, the wrist, and the proximal interphalangeal joint of the index finger, as a function of direction, amplitude, and speed. These peak velocities, pertaining to the extension and flexion dimension of the joints, represent the contribution to the pen-tip displacement of arm, hand, and fingers, respectively. Data of angular velocities in other joint dimensions (not presented here) confirmed the results presented next. 
Direction had a main effect on peak velocities of index finger, $F(1,10)=5.61, p<.05$, and wrist. $F(1,10)=23.97, p<$ .01 . The contribution of the finger was larger in the leftward movements than in the rightward movements, whereas the contribution of the wrist was larger in the rightward movements than in the leftward ones. These results are in agreement with the predicted preferred movement directions for fingers and hand. The arm, which was able to contribute to movements in both directions. contributed most to leftward movements, $F(1,10)=5.07, p<.05$. However, the angular displacement in the elbow must be seen as representing two different activities of the arm, namely, one that translates the forearm along its major axis in leftward movements and another that rotates the forearm around the elbow in rightward movements. Therefore, the importance of the observed effect of direction on elbow excursion should not be overestimated. It is more important to note that the elbow was involved in movements in both directions, particularly when the amplitude of the movement was large. This finding is in agreement with the hypothesis that the forearm is translated or rotated when the fingers or hand alone are not able to achieve a large enough amplitude.

The effects of amplitude on peak velocities of the elbow, $F(1,10)=200.24, p<.01$, and the wrist, $F(1,10)=11.40, p$ $<$.0l, were significant. Both hand and arm had a larger contribution to large movements than to small ones, but the difference between the activities in the two amplitude conditions was larger for the elbow than for the wrist. Although finger angular displacement did not vary significantly with amplitude, in leftward movements it did; the excursion was smaller when amplitude was large than when amplitude was small. A separate ANOVA with an 11 (subject) $\times 2$ (speed) $\times$ 2 (amplitude) design, performed on the data for leftward movements (after averaging across replications) showed that this amplitude effect on leftward movements was significant, $F(1,10)=7.50, p<.05$. These results are in agreement with the predicted distal-to-proximal contribution shift with increased amplitude. The only result that did not confirm this prediction was the fact that, in rightward movements, finger extension and flexion motions (which were very small in this direction anyway) were larger for large amplitudes than for small amplitudes, $F(1,10)=6.84, p<.05$.

Unlike extension and flexion, the contribution of abduction and adduction of the finger (at the metacarpophalangeal joint; not shown in Figure 4) was greater to rightward than to leftward movements, $F(1,10)=11.77, p<.05$, although the contribution was not more than that of finger extension and flexion. However, within these rightward movements, abduction and adduction were not affected by amplitude, $F(1,10)$ $=0.02, p>.05$, either, so the expected decrease of finger involvement with increasing amplitude was seen only in leftward movements.

\section{Effects of Movement Speed on Joint Excursions}

Figure 4 shows that the mean absolute peak angular velocities of elbow, wrist, and finger were a little higher in fast than in slow movements. The mean increases were $32 \%, 23 \%$, and $8 \%$, respectively. These values can be found in Figure 4 as the mean percentage increases across the four direction-amplitude conditions when speed changed from slow to fast. For example, in the bottom panel, increases are shown in elbow peak angular velocity varying between $8.5 \%$ for large left and $53.1 \%$ for small right movements. The average over all four direction-amplitude combinations was the reported $32 \%$. Although increases were found for fingers, wrist, and elbow, the effect was significant for the elbow only, $F(1,10)=6.85, p<.05$. It should be noted that the fact that elbow angular displacement increased significantly, whereas wrist and finger excursions did not, conflicts with the prediction of a contribution shift from highto low-inertia segments as a result of increasing speed.

Mean movement times showed that, in spite of the practice trials they were given, the subjects had trouble obeying the speed instructions. This was probably a result of the fact that, in contrast to the practice trials, during the actual experiment subjects received no feedback on the movement durations they realized. Movement times were $396 \mathrm{~ms}$ for movements that were instructed to be slow and $346 \mathrm{~ms}$ for movements instructed to be fast, whereas the instructed movement times were 400 and $200 \mathrm{~ms}$, respectively. Although, on average, large movements took longer than small movements did ( 437 vs. $306 \mathrm{~ms}$ ), the effect of the speed instruction was equally small in both amplitude conditions. The fact that the observed mean movement times differed only a little for slow and fast movements $(50 \mathrm{~ms}$ instead of the intended $200 \mathrm{~ms}$ ) might be the cause of the nonsignificance of the speed effect for wrist and finger excursions. Therefore, we also performed an analysis on a restricted data set. For each subject and each stroke type, we selected the five fastest trials on which subjects had been instructed to use a fast speed for that stroke type and the five slowest trials on which the speed had to be slow. So, in this data set, a difference in movement duration between slow and fast movements was forced. In this new data set, mean movement time was $512 \mathrm{~ms}$ for slow movements and 274 $\mathrm{ms}$ for fast movements. Speed had a significant effect on elbow, $F(1,10)=37.94, p<.01$, and wrist, $F(1,10)=$ $10.89, p<.01$, excursion but not on angular displacements in the index finger. Elbow contribution was $99 \%$ larger in fast movements than in slow movements, wrist activity was $63 \%$ larger, and finger activity was $20 \%$ larger. So, not only the elbow's contribution increase but also that of the wrist contradicted the hypothesis. We will return to this issue in the Discussion.

\section{Timing of Joint Motions}

Having shown the joint contribution patterns, we now turn to comparisons of the timing of the different joint movements. Figure 5 shows the mean onset, peak, and offset delays of the recorded joint excursions relative to the pen-tip movement. Note that the onset, peak velocity, and offset moments of pen-tip movement were used as reference 


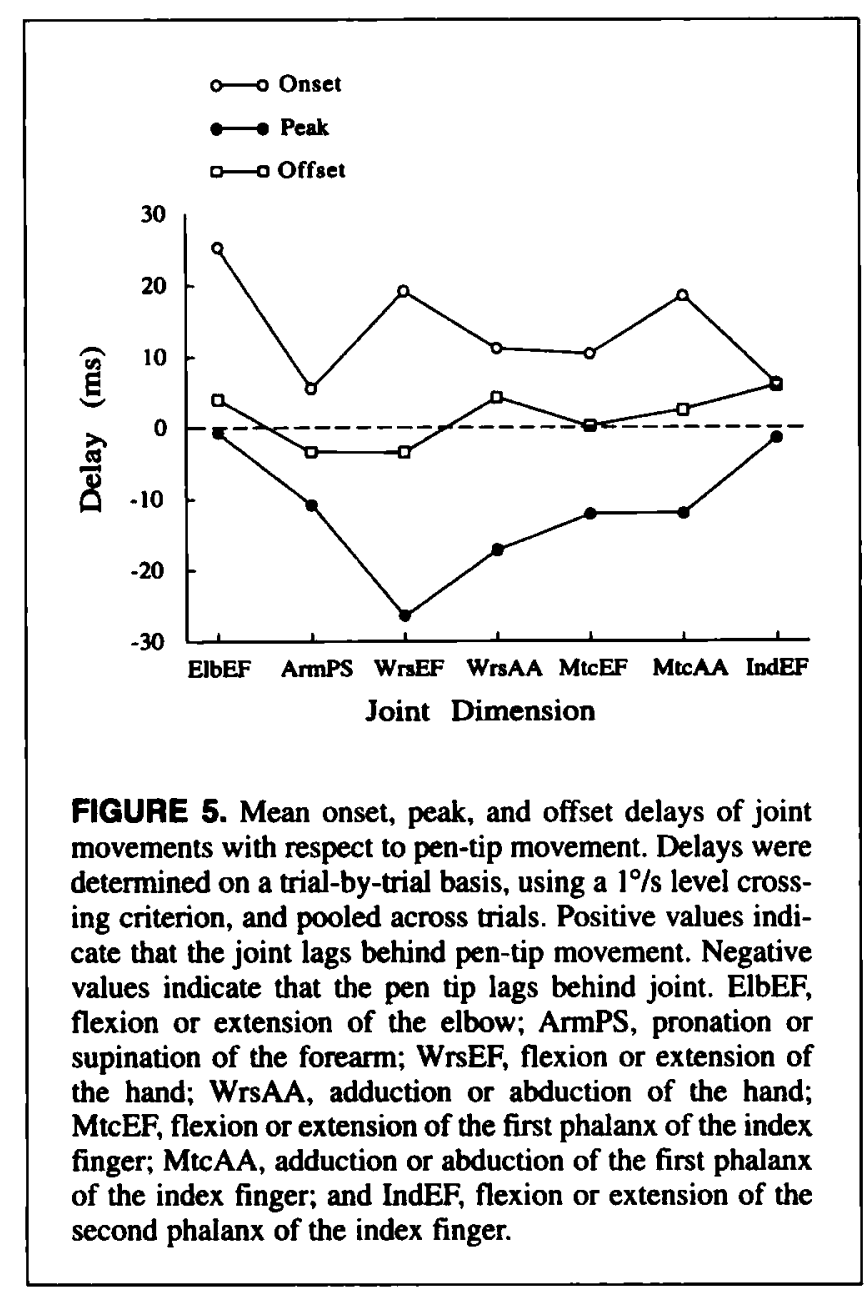

points at the same times for all joints within a movement. The important variables of interest were any between-joints differences in the delays.

Onset delays varied significantly with joint dimension, $F(6,60)=11.19, p<.01$. In other words, the joints did not start moving exactly simultaneously. However, the largest difference between mean start delays was only $20 \mathrm{~ms}$ (between elbow extension and flexion and forearm pronation and supination), which was small in comparison with the mean pen-tip movement time of $371 \mathrm{~ms}$ (5.4\%). Offset delays did not vary significantly with joint dimension, $F(6$, $60)=2.04, p>.05$. The largest difference between offset delays was found between wrist extension and flexion and extension and flexion of the second phalanx of the index finger, and it amounted to $9 \mathrm{~ms}(2.4 \%$ of MT). So, all joints stopped moving at about the same time, approximately simultaneously with the pen-tip movement (mean offset delay $=1.4 \mathrm{~ms}$ ).

The delays of the peak of joint velocity relative to the peak of the pen-tip velocity, were significantly different across joint dimensions, $F(6,60)=4.09, p<.01$. Joint velocities reached their peaks at different times, 1 to $26 \mathrm{~ms}$ prior to the peak in the pen-tip velocity profile. This range of $25 \mathrm{~ms}$ was again small as compared with MT (6.7\%). In short, therefore, considering the relatively small delays between joint movements at their start, peak, and stop, joints moved approximately simultaneously, although there were significant deviations at the start and the peak. To the extent that a principle of synchrony exists, it seems to have been obeyed more strictly at the end of the movement than at the start or at the peak: The variations in offset times were very small and statistically not significant, whereas onset time variations were twice as large and were statistically significant.

These results confirm the data reported by Kaminski and Gentile (1986), in the sense that joints tended to stop at the same time, whereas starting moments varied among joints. However, care should be taken when interpreting our data. One should note that the joint angular velocity profiles were rather variable across trials. Major sources of this variability were inertia and elasticity effects that occurred when the effector came to a halt. Figure 3 shows an example of this. Near the end of the movement in this individual trial, the limb segments were still moving for a short period, coming to a stop in a damped oscillatory fashion, even after the pen tip had stopped moving. Because such dynamical effects were not present at the start of the movement, signal variability was much smaller there. The variability in the signals of individual trials near the end of the movement caused the determination of offset delays to be less reliable, which might explain the asymmetry between start and end delays.

To neutralize variability between individual trials and to view timing at a more general level, we also searched for onset and offset of joint movements in the mean joint angular velocity profiles for each stroke type. These were obtained by averaging time-normalized velocity profiles over the 40 replications of each stroke type by each subject. Time normalization was done after selecting the time interval that consisted of the pen-tip movement time and the immediately preceding and succeeding time intervals with a duration of $25 \%$ of the pen-tip movement time. Thus, the pen-tip movement onset and offset for each trial occurred at exactly one sixth and five sixths of the selected interval, respectively. Note that during time normalization, joint velocity profiles within a trial were not shifted relative to each other. Again, we used a $1 \%$ s level crossing criterion to determine joint movement onset and offset. Figure 6 shows mean joint onset, peak, and offset delays that were obtained in this analysis. After averaging out variability at the end (and, to a smaller extent, at the start) of the joint movements, the mean offset delays were all negative, ranging from $-3.2 \%$ to $-14.7 \%$ of MT. So, the largest delay between the offset of movements in different joint dimensions was $11.5 \%$ of MT. This is comparable with the largest difference between onset delays (largest difference, $9.8 \%$ of MT). Again, these delays were small, relative to movement time. However, onset, peak, and offset delays varied significantly with joint dimension: There were significant main effects of joint dimension on onset, $F(6,60)=2.88, p<.05$, peak, 


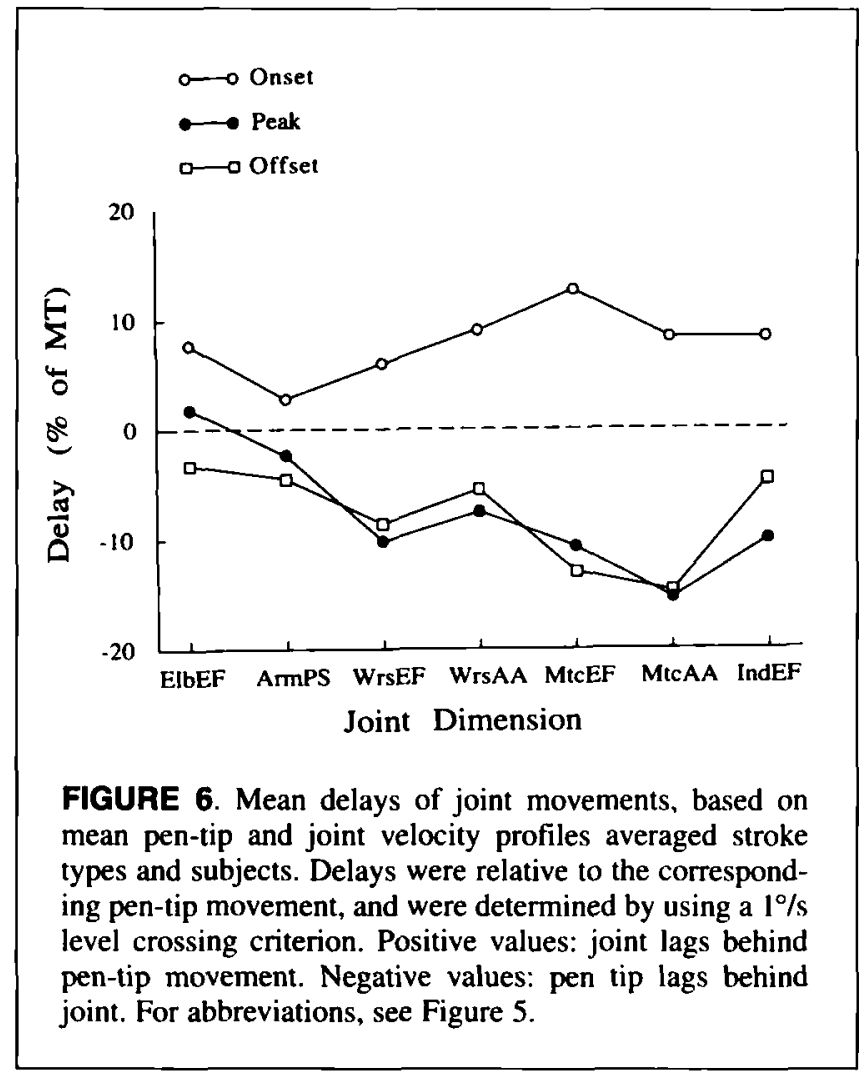

$F(6,60)=4.47, p<.01$, and offset delays, $F(6,60)=5.29$, $p<.01$. Therefore, when variations in individual trials were neutralized, deviations from synchrony appeared also at movement termination. Although the deviations were again relatively small, one can conclude that joints do not move exactly simultaneously.

A possible explanation for the small but significant deviations from synchrony between movements in different joint dimensions might be suggested by the following observation. If two velocity profiles are congruent, the one with the largest peak height will rise above a certain absolute level earlier in time than the one with the lowest peak height. So the observed variations in the onset and offset delays (which are, in fact, determined by level crossings), might be explained by different peak heights in the joint velocity profiles (see Teasdale, Bard, Fleury, Young, \& Proteau, 1993). Indeed, we found, in 7 of the 11 subjects, significant negative correlations between peak height and onset delays (based on the average velocity profiles for each condition and subject). These data indicate that onset delays between joint movements may, at least partly, be accounted for by peak velocity differences in combination with the fixed-level crossing criterion. In contrast, correlations between peak height and offset delays were never significant, possibly because near movement offset the mean velocity profiles showed small deviations from the bell shape (see Figure 7).

Kaminski and Gentile (1986) found that in horizontal planar arm movements, the elbow and shoulder did not start moving at the same time but did stop simultaneously. More precisely, the joint moving through the largest angle was found to start moving before the other joint. The relation between angle difference (reflecting contribution difference) and onset difference was strongly linear. The data from the present experiment showed correlations between peak height and onset delay that were in line with these findings, but one cannot rule out the possibility that this relation was induced by the fixed-level criterion for onset determination, as explained in the previous paragraph.

Summing up our findings concerning timing, the results suggest that joints do not move completely simultaneously. However, the deviations from synchrony are small at the start and at the peak as well as at the end of the movement. Moreover, the occurrence of these deviations at movement onset may partly be explained by the combined effect of the joints' peak velocity and the level crossing criterion.

\section{Characteristics of the Observed Velocity Profiles}

To illustrate a few general aspects of the joint movements observed in the experiment, in Figure 7 we show mean joint angular velocity profiles obtained after time normalization and averaging across subjects and replications of all trials in one of the eight experimental conditions (fast, large movement to the right). The figure shows, first of all, that the joints that were most involved in a certain condition (in this case, elbow and wrist) had velocity profiles that were essentially unimodal and resembled bell shapes. Joints with lower peak angular velocities (finger joints) had mean profiles that may have been bimodal with low peak velocities. These relatively irregular mean velocity profiles reflected the instability of the motion that was observed in individual trials for joints that had a relatively small contribution to the movement (see also Figure 3). Furthermore, joints came to a halt in a damped oscillatory fashion (only a part of the first period of these oscillations is shown here). As a result, there were deviations from the bell shape near the end of the movement, even for significantly contributing joints. Near the start of the movement, deviations from bell shape were less pronounced. Although these observations applied to most of the joint excursions that were recorded, pronation and supination movements of the forearm behaved differently. In all conditions, multimodal velocity profiles were seen, whereas peak velocity varied with the conditions. In Figure 7 , for example, one can see that peak velocity of the forearm was as high as those for the wrist and elbow were, which indicates large angular displacements in this condition. Yet, the profile deviated substantially from a bell shape. Indeed, inspection of individual trials shows that, in general, the motion in this particular joint dimension was multimodal. We will return to this observation in the Discussion section.

\section{Discussion and Conclusions}

In this study, we investigated limb segment involvement as a function of movement direction, amplitude, and speed in a graphic pointing task. Other studies in which both end-effec- 

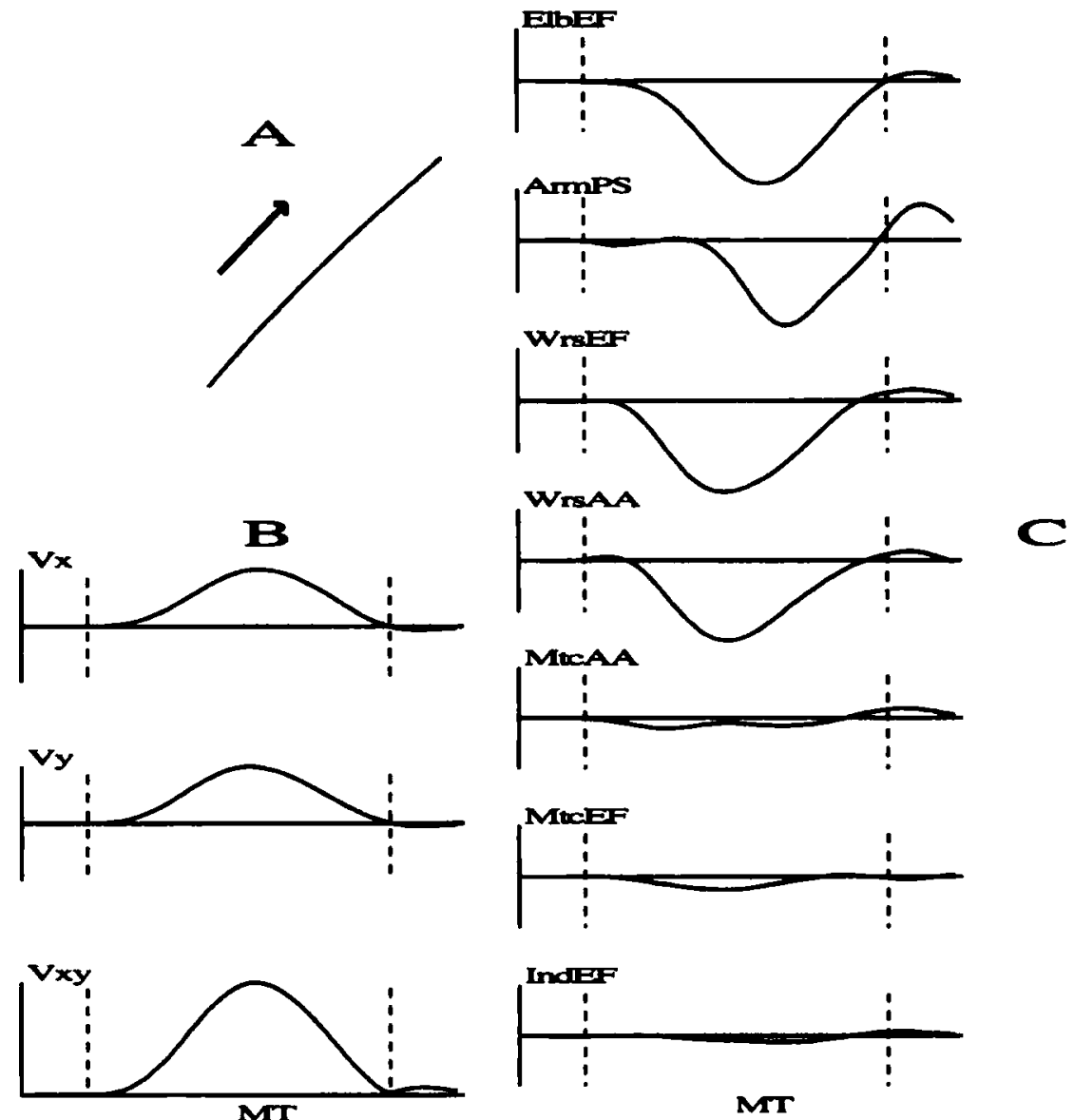

FIGURE 7. Mean pen-tip path and velocity profiles for all joint excursions, for fast, large, rightward movements, averaged across subjects and replications. A. Pen-tip path. B. Corresponding tangential velocity profile $(\mathrm{V} x y)$ and the horizontal and vertical velocity profiles $(\mathrm{V} x$ and $\mathrm{Vy}$ ). C. Angular velocity profiles of all recorded joint excursions. The dotted lines in the velocity profiles delimit the interval in which the main stroke was produced. Positive angular velocities indicate flexion, negative velocities indicate extension. The arrow indicates pen-tip movement direction. Before averaging, the velocity profiles were time normalized such that pen-tip movement time was equal for all trials. Depicted is $25 \%$ of MT before pentip movement, $100 \% \mathrm{MT}$, and $25 \%$ of MT after pen-tip movement. Ordinate scales are equal for all joint velocity profiles and are counted in arbitrary units. The same holds for the pentip velocity profiles. For abbreviations, see Figure 5.

tor and joint kinematics were investigated in relation to these parameters mainly investigated continuous movement performance, such as back-and-forth movements (Meulenbroek et al., 1993), circle drawing, or handwriting (Lacquaniti et al. 1987; Lacquaniti, 1989). It was our intention to investigate to what extent the results that were observed in such cyclic movements also apply to discrete movements.

We found that the selection of limb segment involvement patterns during the execution of the discrete movements in that task depended on the suitability of the limb segments to produce movements along the required movement axis and on their optimal amplitude of motion. With respect to direction of movement, we found that the contribution of the finger was largest in movements in the upper left direction, whereas the hand had its largest contribution in movements in the upper right direction. The arm was involved in movements in both directions. These results confirmed earlier findings concerning the effect of movement direction on limb segment involvement in cyclical drawing movements (Meulenbroek et al., 1993).

Regarding amplitude, we found that both hand and arm excursions were larger in large movements than in small ones, whereas finger excursions tended to be smaller. These results agree with findings of Meulenbroek et al. (1993), who showed a similar effect of amplitude in cyclical drawing. We also found effects of movement speed on limb segment involvement. However, the effects differed from what we expected. As movement speed increased, the proximal 
limb segments became more involved. Our hypothesis was that the larger, proximal segments become less involved as speed demands increase. This hypothesis was based on findings that the optimal movement frequency is lower for large, heavy limb segments (proximal) than for smaller and lighter (distal) parts of the limb (Rosenbaum et al., 1991). A possible explanation for the unexpected results, may be that, in a drawing task, synergies may be formed that are different from the ones formed in the fingertip oscillation task used by Rosenbaum et al. (1991). Whereas in the latter study there was no coupling whatsoever (subjects merely had to wave a finger through the air), in the present drawing task the constraint of having to hold the pen between thumb and index finger demanded a tight coupling of these limb segments. As a result, the optimal frequencies of the synergies in both tasks may have differed. However, even though, especially for the fingers, there may have been essential differences in synergy formation between the two tasks, the earlier results of Meulenbroek et al. (1993) show that even in a normal writing posture, effects of optimal frequencies on limb segment selection can be observed. So the unexpected speed effects cannot be explained solely by differential synergy formation in drawing and writing, as opposed to oscillating a finger.

In cyclical movements, minimal energy expenditure may be achieved by having all involved limb segments oscillate with a frequency that is close to the average of the resonant frequencies of each segment. According to Rosenbaum et al. (1991), this is why large and heavy limb segments are selected when movement frequencies are low, whereas smaller and lighter segments are selected when movement frequencies are high. However, the absence of oscillation in discrete movements may make the benefit of a fit between resonant frequency and task demands as a criterion for the selection of limb segments less pronounced. As a result, other constraints related directly to the discrete character of the task may outweigh it as a coordination principle. In general, continued cyclic movements may allow for a more efficient exploitation of biomechanical characteristics of the effector system than isolated, discrete movements do. In the latter movement type, therefore, the focus could be on obeying the task demands as much as possible, even at the expense of increased movement costs. For example, faster movements could be made by increasing involvement of the limb segments served by the shortest neural circuits (proximal joints), or of those with the most powerful muscles, instead of those with high resonant frequencies.

As far as the timing of limb segment movements is concerned, we found that there were significant differences in movement onset and offset times for the various limb segments. Although the deviations from synchronous movement onset and offset were small as compared with the duration of the movements, the observations of onset differences were not clearly in contrast with those reported by Kaminski and Gentile (1986). Still, the cause of the onset and offset deviations that we observed is not completely clear. Do they originate from a certain coordination strategy, or are they a result of mechanical aspects of the movement such as, for example, friction with the writing surface or skin deformations influencing the measurements? Considering the fact that the differences were small and that they were not convincingly larger for onset than for offset, we feel that (in the absence of requirements concerning the shape of the end-effector path) limb segment movements are probably planned to be executed simultaneously. Influences of a biomechanical or physical nature may cause the observed timing to deviate from the intended timing. A strategy involving simultaneous joint motions provides a simple coordination mechanism that does not require a complex timing method. The possible disadvantage of having limb segments move with nonpreferred speeds so that the required angular displacement can be produced may be nullified by the advantage of not having to determine the moment that each limb segment's movement starts and stops. In a sense. this may be seen as a kind of coupling, reducing the number of degrees of freedom at the timing level.

Elimination of degrees of freedom as a coordination strategy may also underlie the characteristics of the joint angular velocity profiles. In general, mean velocity profiles were found to resemble bell shapes, at least for the joints that contributed most in a trial. However, for less involved joints in a trial, and for pronation and supination in general, profiles were more variable. We feel that this variability may be of a reactive nature. Considering pronation and supination, the observed motion could result from the constraint of having to keep the pen tip on the writing surface: As fingers, hand, and arm move the pen in a certain direction, pen-tip pressure may change in response to the compliance requirement and cause the forearm to rotate around its longitudinal axis. Inversely, one may need pronation and supination to hold the pen down when the motion in other joints causes the pen tip to rise. Similar effects may cause other joints that do not have a crucial contribution to the movement to also react. Such reactive joint activity could be used as part of a coordination principle that holds that one explicitly plans the movement of certain limb segments to perform the main aspects of the required task, whereas the motion of other segments is not explicitly planned but just follows the principal movement. This has the advantage of reducing the number of degrees of freedom that have to be taken into account during the planning process.

The present experiment showed that in discrete movement tasks, common movement initiation and termination moments of joint excursions are violated, albeit only to a small extent. This is in contrast to cyclical movement tasks in which maintaining constant phase relationships between the neighboring joints of a linear effector system (Lacquaniti, 1989; Soechting \& Lacquaniti, 1981; Soechting et al., 1986) seems to be an efficient control principle. However, it is extremely difficult to attribute our findings either to kinematic invariants of central planning processes or to dynamic consequences of motion that only emerge during 
execution as a result of interactions between the moving effector system and the environment (Hasan, 1991). Deviations from unimodal bell-shaped velocity profiles were frequently observed in the present experiment in the pronation and supination of the forearm and in joints that, in a particular movement, seemed hardly to contribute. Nevertheless, an absolute velocity criterion of $1 \% \mathrm{~s}$ resulted in joints' starting and stopping almost simultaneously with the beginning and end of the pen-tip displacement. The general picture that emerges from the data is that movement direction in extrinsic space stands out as the independent variable that most consistently determines the limb segment configuration to be recruited to perform the task and the limb segments that will be involved if subsequent amplitude or speed requirements pose constraints on the direction-dependent organization. This induces either a parametric change of the recruited joints or, if necessary, the additional recruitment of a different effector configuration. This points to a hierarchical control of limb segment coordination, with movement direction having a dominant role in the recruitment process, a view that corresponds to recent neurophysiological evidence regarding the strong correlation between movement direction and the pooled activity of motor cortex neurons, expressed in terms of vectors (Georgopoulos, 1990; Georgopoulos, Schwartz, \& Kettner, 1986; Schwartz, 1994). Because we investigated only a small number of conditions, further parametric studies are needed that describe the changes of joint kinematics as a function of the variables presently investigated more precisely.

\section{ACKNOWLEDGMENT}

The research of R. G. J. M. has been made possible by a fellowship from the Royal Netherlands Academy of Arts and Sciences

\section{NOTES}

1. In earlier studies (Meulenbroek et al., 1993; Rosenbaum et al. 1991), the relative contribution of a limb segment to a pen-tip movement, expressed as the percentage of pen-tip displacement that could be attributed to that limb segment's angular displacement, was studied. The length of each limb segment influenced the contribution observed for that limb segment. In the present work, we studied the limb segments' involvement by measuring the absolute peak velocity of their angular displacements, instead of the amount of pen-tip displacement that could be attributed to their involvement. This was done because we were primarily interested in the effects of the experimental conditions on the involvement of the individual joints. Moreover, it turned out to be very hard in our experimental set-up to estimate the net effect of a joint excursion on pen-tip displacement.

2. When we speak about noise in the context of angular displacements and velocities, we are primarily referring to noise related to the sampling process as well as artifacts introduced during the process of filtering and finding derivatives. In addition to this noise, some artifacts may have been caused by IRED displacements that resulted from soft tissue deformations as a result of the movement. Although IRED locations were chosen such that these artifacts were minimal, they could not be ruled out completely. These artifacts are referred to as measurement error.

\section{REFERENCES}

Bernstein, N. (1967). The coordination and regulation of movement. London: Pergamon.

Cruse, H., Brüwer, M., \& Dean, J. (1993). Control of three- and four-joint arm movement: Strategies for a manipulator with redundant degrees of freedom. Journal of Motor Behavior, 25, 131-139.

Fitts, P. M. (1954). The information capacity of the human motor system in controlling the amplitude of movement. Journal of Experimental Psychology, 47, 381-391.

French, A. P. (1971). Vibrations and waves. New York: W. W. Norton.

Georgopoulos, A. P., (1990). Neurophysiology of reaching. In M. Jeannerod (Ed.), Attention and performance XIII (pp. 227-263). Hillsdale, NJ: Erlbaum.

Georgopoulos, A. P., Schwartz, A. B., \& Kettner, R. E. (1986). Neuronal population coding of movement direction. Science, 233, 1416-1419.

Guiard, Y. (1993). On Fitts's and Hooke's laws: Simple harmonic movement in upper-limb cyclical aiming. Acta Psychologica, $82,139-159$.

Hasan, Z., (1991). Biomechanics and the study of multijoint movements. In D. R. Humphrey \& H.-J. Freund (Eds.), Motor control: Concepts and issues. New York: Wiley.

Kaminski, T., \& Gentile, A. M. (1986). Joint control strategies and hand trajectories in multijoint pointing movements. Journal of Motor Behavior, 18, 261-278.

Kay, B. A., Kelso, J. A. S., Saltzman, E. L., \& Schöner, G. (1987). Space-time behavior of single and bimanual thythmical movement: Data and limit cycle model. Joumal of Experimental Psychology: Human Perception and Performance, 13, 178-192.

Lacquaniti, F. L. (1989). Central representations of human limb movement as revealed by studies of drawing and handwriting. Trends in Neurosciences, 12, 287-291.

Lacquaniti, F., Ferrigno, G., Pedotti, A., Soechting, J. F., \& Terzuolo, C. (1987). Changes in spatial scale in drawing and handwriting: Kinematic contributions by proximal and distal joints. The Journal of Neuroscience, 7, 819-828.

Meulenbroek, R. G. J., Rosenbaum, D. A., Thomassen, A. J. W. M., \& Schomaker, L. R. B. (1993). Limb-segment selection in drawing behavior. The Quarterly Joumal of Experimental Psychology, 46A, 273-299.

Meulenbroek, R. G. J., \& Thomassen, A. J. W. M. (1991). Strokedirection preferences in drawing and handwriting. Human Movement Science, 10, 247-270.

Rosenbaum, D. A., Loukopoulos, L. D., Meulenbroek, R. G. J., Vaughan, J., \& Engelbrecht, S. E. (1995). Planning reaches by evaluating stored postures. Psychological Review, 102, 28-67.

Rosenbaum, D. A., Slotta, J. D., Vaughan, J., \& Plamondon, R. (1991). Optimal movement selection. Psychological Science, 2, 86-91.

Schwartz, A. B. (1994). Direct cortical representation of drawing. Science, 265, 540-542.

Soechting, J. F., \& Lacquaniti, F. (1981). Invariant characteristics of a pointing movement in man. The Journal of Neursscience, $1,710-720$.

Soechting, J. F., Lacquaniti, F., \& Terzuolo, C. A. (1986). Coordination of arm movements in three-dimensional space. Sensorimotor mapping during drawing movement. Neuroscience, 17, 295-311.

Teasdale, N., Bard, C., Fleury, M., Young, D. E., \& Proteau, L. (1993). Determining movement onsets from temporal series. Journal of Motor Behavior, 25, 97-106.

Turvey, M. T. (1990). Coordination. American Psychologist, 45, 938-953. 
Van Emmerik, R. E. A., \& Newell, K. M. (1990). The influence of task and organismic constraints on intralimb and pen-point kinematics in a drawing task. Acta Psychologica. 73, 171-190.

\section{APPENDIX}

\section{Derivation of Joint Angles}

In this Appendix, we explain the derivation of the joint angles from the location of the 14 IREDs attached to the subject's arm. The IREDs constituted a number of vectors and planes in space. Three-dimensional angles between these vectors and planes were used as an estimate of the seven joint angles, as described below.

The following notation was used:

* $(a, b)$ indicated the direction vector of the line between IREDs $a$ and $b$.

$*(a, b, c)$ indicated the plane (of infinite size) defined by IREDs $a, b$, and $c$.

IREDs were numbered as shown in Figure 2.

Flexion or extension of the elbow: The smallest angle between
$(1,3)$ and $(3,6)$.

Pronation or supination of the forearm: $-90^{\circ}$ plus the smallest angle between $(7,6)$ and the normal vector of $(1,3,2)$.

Abduction or adduction of the hand: $90^{\circ}$ minus the smallest angle between $(9,10)$ and the projection of $(4,5)$ on the hand plane $(8,9,10)$.

Extension or flexion of the hand: $90^{\circ}$ minus the smallest angle between $(4,5)$ and the normal vector of $(8,9,10)$.

Abduction or adduction of the first phalanx of the index finger: $-90^{\circ}$ plus the smallest angle between $(9,10)$ and the projection of $(11,12)$ on the hand plane $(8,9,10)$.

Extension or flexion of the first phalanx of the index finger: The angle between $(11,12)$ and its projection on $(8,9,10)$.

Extension or flexion of the second phalanx of the index finger: $90^{\circ}$ minus the angle between $(11,12)$ and $(13.14)$.

Submitted February 6, 1995

Revised May 23, 1995 